\title{
Global Dynamics of Shaft Lines Rotating in Surrounding Fluids Application to Thin Fluid Films
}

\author{
David Lornage and Georges Jacquet-Richardet \\ INSA Lyon, Laboratoire de Mécanique des Structures, Villeurbanne, France
}

While often sufficiently accurate, approaches using rotor dynamics and bladed disc dynamics are not adapted to the study of certain important cases, i.e., when observing wheel/shaft coupling or when fluid elements are strongly coupled with local structural deformations. The approach proposed here is a step toward a global model of shaft lines. The whole flexible wheel/shaft assembly and the influence of specific fluid film elements are considered in a full threedimensional model. A modal projection associated with a grid located at the interface of the fluid and structural domains provides an efficient and adaptable coupling. The equations governing the whole system are solved within a time marching procedure which alternatively considers the equations of fluid and structure. The technique chosen is applied to two different test cases. The first is composed of a disc and a thin-walled shaft mounted on a hydrodynamic bearing. The second is intended for studying a more realistic structure composed of a shaft and a wheel coupled with a fluid film between the wheel and a casing. These applications make it possible to identify trends related to fluid effects and couplings between the flexible structural parts.

Keywords Fluid film, Fluid/structure coupling, Interfacing grid, Modal reduction, Shaft lines dynamics

High performances, reliability, and safety require a highly accurate prediction of turbomachine behavior. In this context, the work proposed aims at developing a global model of wheel/shaft assemblies rotating in surrounding fluids.

Two uncoupled approaches are generally used to predict the dynamic behavior of rotating parts of machines.

Received 22 January 2003; accepted 30 June 2003.

Address correspondence to Georges Jacquet-Richardet, INSA Lyon, Laboratoire de Mécanique des Structures, 8 rue des Sciences, 69621 Villeurbanne, France. E-mail: georges.jacquet@insa-lyon.fr

1. The rotor dynamics approach deals with global shaft behavior in bending/torsion (in this case wheels are supposed to be rigid).

2. The bladed disk assembly dynamics approach concerns isolated wheels.

Considering rotor dynamics, the general effect of fluid films has been the subject of many studies, mainly focusing on hydrodynamic seals, journal bearings, and thrust bearings. Fluid films have a major influence on the global behavior of shaft lines, since they add stiffness and damping and govern the stability of the system (Frêne et al., 1990; Iwatsubo et al., 1990). Leakage flows between wheel (flanges) and casing are also very important and govern stability (Childs, 1991). Other studies deal with shafts rotating in a fluid and shafts rotating with a fluid inside (Baskarone and Hensel, 1991; Berlioz et al., 1996).

Concerning bladed disk assembly dynamics, fluid/structure problems are divided into two main types. The first group deals with the dynamic behavior of structures rotating in heavy fluids and considers added mass and damping effects. The second concerns light fluids and flutter instability (Bendiksen, 2000; Moyroud, 1998) and the air film effect on rotating disc (computers) behavior (Renshaw, 1998).

To model these problems, the most rigorous coupling technique lies in considering fluid and structural domains in the same numerical scheme. This technique, mainly developed in the context of aeroelasticity (Bendiksen, 2000), has major drawbacks in this case, since it requires developing very specific and cumbersome tools. On the other hand, very weak coupling methods have been developed based on independent fluid and structural computations. Between these two extremes, intermediate coupling techniques involve solving alternatively fluid and structural models within a stepped information transfer process. If used with sufficient accuracy, these coupling techniques do not lead to information loss. Moreover, they present major advantages. They use existing models, validated independently by specialists in each field and they also lead to general and openended tools since model substitutions are possible without major modifications. 
Taking into account the progression of machines and the performances required of them, many configurations can only be solved by using these types of global models. For example, wheel/shaft couplings can have a significant influence on shaft lines behavior (Jacquet-Richardet et al., 1996; Irretier et al., 1999). Concerning fluid films, their behavior is sensitive to defects or local deformations. For example, Berger et al. (2000) showed that the dynamic behavior of rotor/hydrodynamic thrust bearing assemblies differs significantly whether taking thrust bearing defects into account or not. Desbordes et al. (1994) showed the influence of pad elastic deformations on the trajectory of a shaft mounted on an oscillating pads bearing. Lornage et al. (2001) showed the influence of local disc deformations on the global behavior of a simple disc/shaft assembly coupled with a thin fluid film between the disc and a casing.

This article first describes the procedure proposed, adapted to a global modeling of the dynamic behavior of shaft lines rotating in surrounding fluids after which it is applied to two test cases. The first test case is composed of a simple disc/thin walled shaft assembly mounted on a hydrodynamic bearing. The second is a more realistic wheel/shaft assembly coupled with a leakage flow between the wheel and a casing.

\section{NUMERICAL MODEL}

\section{Structural Model}

Complex shaped nonaxisymmetric structures are discretized by using the finite element method and studied with respect to the rotating frame. The equations, governing the motion of the structure rotating in a fluid environment, are derived from the internal energies (kinetic, potential, dissipation) as well as from the work done by the external forces, by applying Lagrange's equations. The system obtained is the following (Jacquet-Richardet et al., 1996):

$$
\begin{gathered}
{[M]\{\ddot{\delta}\}+\left[C+C_{n}+C_{r}\right]\{\dot{\delta}\}+\left[K_{E}+K_{S}+K_{G}+K_{A}+K C_{n}\right]} \\
\{\delta\}=\{F(\delta, \dot{\delta}, \ddot{\delta})\}
\end{gathered}
$$

where $\{\ddot{\delta}\},\{\dot{\delta}\}$, and $\{\delta\}$ are the nodal acceleration, velocity, and displacement vectors, respectively. $[M]$ and $\left[K_{E}\right]$ are the standard mass and elastic stiffness matrices. [C], $\left[K_{S}+K_{G}+K_{A}\right]$ are linked to the effect of rotation, $\left[C_{n}+C_{r}\right]$ and $\left[K C_{n}\right]$ are matrices accounting for damping, and $\{F\}$ is the nodal forces vector. The effects of rotation are divided into gyroscopic effects $[C]$, stress stiffening $\left[K_{G}\right]$, and spin softening $\left[K_{S}\right]$ effects, and into effects induced by rotational speed variations $\left[K_{A}\right]$. Two types of damping are considered: internal or rotating damping, $\left[C_{r}\right]$, and nonrotating damping, $\left[C_{n}\right]$ and $\left[K C_{n}\right] .\{F\}$ accounts for classical forces (weight, unbalance, etc.) as well as forces induced by the fluid.

\section{Fluid Model}

The procedure proposed can be used whatever the fluid model. However, in order to keep computational effort at an acceptable level, applications have been limited to cases involving thin fluid films where inertial effects are neglected and the flow is laminar. For an incompressible fluid, located between two walls with no effect of temperature, the pressure in the film is governed by the classical Reynolds equation. In a Cartesian frame, this equation is written as:

$$
\begin{aligned}
\frac{\partial}{\partial x_{1}}[ & \left.\frac{\left(H_{2}-H_{1}\right)^{3}}{\mu} \frac{\partial p}{\partial x_{1}}\right]+\frac{\partial}{\partial x_{3}}\left[\frac{\left(H_{2}-H_{1}\right)^{3}}{\mu} \frac{\partial p}{\partial x_{3}}\right] \\
= & 6 \frac{\partial}{\partial x_{1}}\left[\left(U_{11}+U_{21}\right)\left(H_{2}-H_{1}\right)\right]-12 U_{21} \frac{\partial H_{2}}{\partial x_{1}} \\
& +12 U_{11} \frac{\partial H_{1}}{\partial x_{1}}+6 \frac{\partial}{\partial x_{3}}\left[\left(U_{13}+U_{23}\right)\left(H_{2}-H_{1}\right)\right] \\
& -12 U_{23} \frac{\partial H_{2}}{\partial x_{3}}+12 U_{13} \frac{\partial H_{1}}{\partial x_{3}}+12\left(U_{22}-U_{12}\right)
\end{aligned}
$$

where $\mu$ is the fluid dynamic viscosity, $\mathrm{h}$ is the film thickness, and $p$ is the pressure in the fluid. $U_{i}, V_{i}, W_{i}(i=1,2)$ are, respectively, wall velocities in the $x, y$, and $z$ directions.

Equation (2), associated with the Reynolds boundary conditions, is solved using finite differences associated with a GaussSeidel iterative process with over relaxation (Frêne et al., 1990).

\section{Fluid Structure Coupling and Solution}

A direct solution of system (1) gives major drawbacks. Firstly, when considering real structures, accurate analysis requires a large number of degrees of freedom, leading to prohibitive computer costs. Secondly, fluid effects must be transferred into nodal equivalent forces, generally leading to information losses. Thirdly, a full fluid and structural model, using compatible meshes and numerical procedures, is often not desirable as it supposes the use of very specific tools. As shown below, these drawbacks can be overcome by considering a modal approach coupled with an interfacing grid concept.

\section{Modal Reduction}

Let us consider the undamped structure at rest in a vacuum. Thus, in this case, (1) reduces to:

$$
[M]\{\ddot{\delta}\}+\left[K_{E}\right]\{\delta\}=\{0\}
$$

The solution of this system, done directly or by using a cyclic symmetrical approach, gives a set of mode shapes $\left\{\Phi_{i}\right\}$, which can be grouped into a modal matrix $[\Phi]$. Assuming that the unknown displacements $\{\delta\}$ can be written as a linear combination of these basic shapes, then:

$$
\{\delta\}=[\Phi]\{q\}
$$

and system (1) becomes:

$$
[m]\{\ddot{q}\}+[c]\{\dot{q}\}+[k]\{q\}=\{f\}
$$

where $[\mathrm{m}],[\mathrm{c}]$, and $[\mathrm{k}]$, respectively, full modal mass, damping, and stiffness matrices and $\{\mathrm{f}\}$, the modal forces vector 
disregarding forces induced by the fluid, are given by:

$$
\begin{aligned}
{[m] } & =[\Phi]^{t}[M][\Phi] \\
{[c] } & =[\Phi]^{t}\left[C+C_{n}+C_{r}\right][\Phi] \\
{[k] } & =[\Phi]^{t}\left[K_{E}+K_{S}+K_{G}+K_{A}+K C_{n}\right][\Phi] \\
\{f\} & =[\Phi]^{t}\{F\}
\end{aligned}
$$

In addition to the reduction in computational effort, the modal approach gives two other important advantages. Firstly, modal quantities can be obtained using different original meshes and numerical schemes. Secondly, structural damping can be introduced in its modal form by using, for example, experimental results. This reduction has been validated with respect to numerical and experimental reference results in Jacquet-Richardet et al. (1996).

\section{Fluid-Structure Coupling and Solution}

The structure is discretized by using finite elements. For the fluid domain, various discretization techniques are used classically: finite elements, finite volumes, finite differences, and the mesh is often very fine (much more than the structural mesh). Consequently, the two meshes are not usually compatible at the interface, with respect to both the type of discretization technique and the mesh density. An interface grid concept is used to maintain fluid and structure mesh properties.

Modal quantities are physical quantities and are thus independent of the mesh used, provided the latter is fine enough. Consequently, if mode shapes and fluid-induced pressures are known on a grid, situated at the interface between the two domains but independent from the original meshes, the modal forces induced by the fluid can be obtained simply from:

$$
\{f\}=\left[\Phi_{I}\right]^{t}\left\{F_{I}\right\}
$$

where $\left[\Phi_{I}\right]$ is the projection of mode shapes onto the interface grid and $\left\{F_{I}\right\}$ is the nodal equivalent forces calculated from the pressure field, taking into account the intrinsic shape functions of the interface grid.

The interface grid considered is constituted with planar fournoded finite elements. It is linked to the undeformed interface, rotates with the structure, and is as fine as the finest mesh, which is usually the fluid mesh. This grid gives obvious advantages in terms of efficiency because, in this case, basic meshes can be constructed independently, considering only the convergence requirements of each domain. It also gives advantages in terms of flexibility, because for coupling, nothing needs to be known concerning the intrinsic characteristics of the original elements used. The only information needed is the location of nodes and the associated nodal displacements for the structure and pressures for the fluid, information which is easily available when using existing codes.

The coupling process implies several transfers between the original and interface grids:
1. Mode shapes from the structural mesh $\left[\Phi_{S}\right]$ to the interface grid $\left[\Phi_{I}\right]$.

2. Structural displacements and velocities, calculated using $\left[\Phi_{I}\right],\{q\}$, and $\{\dot{q}\}$ according to Equation (4), from the interface grid to the fluid mesh.

3. Pressures from the fluid mesh to the interface grid.

The first transfer is performed only once and is quite simple as it concerns data with smooth spatial variations. The two other transfers must be performed for each time step but can be simple if the original fluid mesh is consistent with the interface grid. A full description of the transfer algorithm can be found in Moyroud et al. (2000).

Equation [5] is solved using the Runge-Kutta's timemarching procedure of the fourth order. Very efficient for finding the numerical solution of differential equations, this procedure is generally quite stable and accurate. For linear systems, the time step $\Delta t$ used must verify $\Delta t<1 / 10 f_{\max }$, where $f_{\max }$ is the highest frequency of the mode shapes retained in the modal basis. However, due to the highly nonlinear property of hydrodynamic forces, the time step considered for the application is $\Delta t=10^{-6} \mathrm{~s}$, a value significantly lower than the classical limit $\left(1 / 10 f_{\max }=3 \cdot 10^{-5} \mathrm{~s}\right)$.

The main steps of the solution technique are summarized in Figure 1. The dependency of the matrices upon rotational speed is fully taken into account at each time step. For the application, where the effect of stress stiffening is not significant, the associated matrix has been linearized.

\section{APPLICATIONS}

The first test case deals with a well-known configuration composed of a disc/shaft assembly mounted on a hydrodynamic bearing. Applied to a thin-walled shaft, the main goal of this

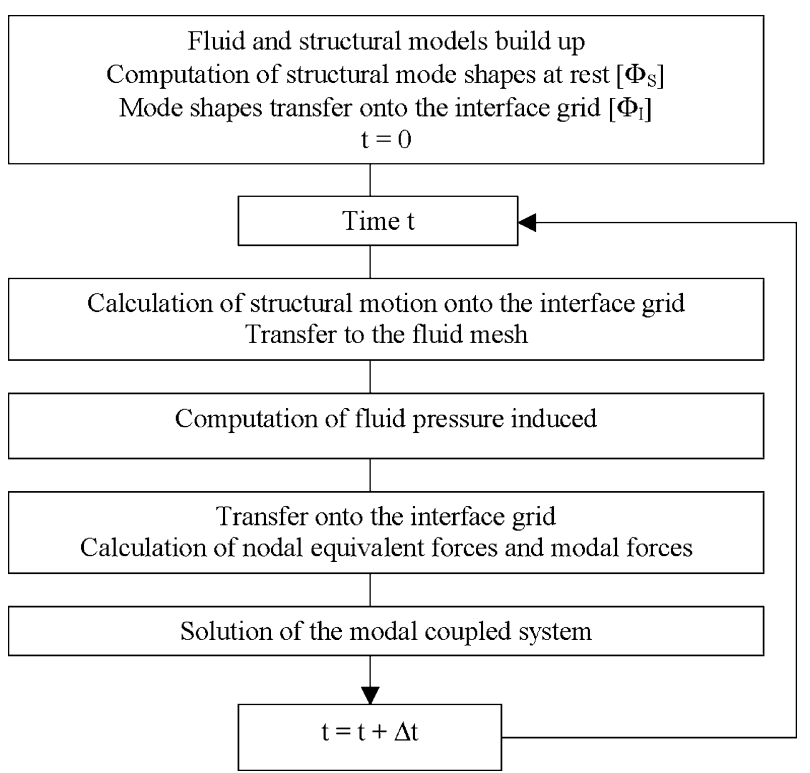

FIGURE 1

Solution procedure. 


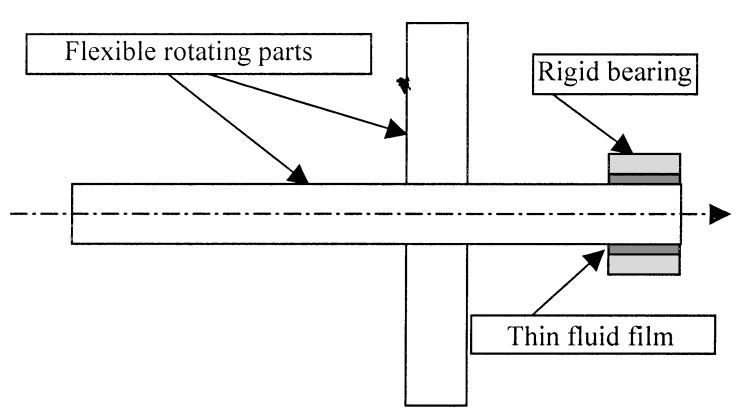

FIGURE 2

Coupled disc/shaft-bearing system.

part is to examined the influence of section deformations and to study the influence of the number and the nature of the modes retained in the modal basis. The second application, while remaining academic, is more realistic. It concerns a wheel/shaft assembly coupled with a fluid film located between one of the wheel flanges and a casing.

\section{Test Case $\mathbf{N}^{0} \mathbf{1}$}

\section{Presentation}

The structure is composed of a thin-walled shaft and a disc mounted at $70 \%$ of shaft length (Figure 2). The dimensions of the shaft are the following: length $L=0.65 \mathrm{~m}$, outer radius $R_{e}=0.04 \mathrm{~m}$, and thickness $e_{s}=0.001 \mathrm{~m}$. The disc radius is $R_{d}=0.2 \mathrm{~m}$ and its thickness is $e=0.02 \mathrm{~m}$. Both shaft and disc are made of steel (Young's modulus $E=2.10^{11} \mathrm{~Pa}$, density $\rho=7800 \mathrm{~kg} / \mathrm{m}^{3}$ ). The shaft is clamped at one end and supported by a hydrodynamic bearing at the other end. The radial clearance and the length of the bearing are $C=0.1 \mathrm{~mm}$ and $l=0.02 \mathrm{~m}$, respectively. The fluid dynamic viscosity is $\mu=0.01$ Pa.s.

The rotor is considered under constant rotation at $\Omega=$ $10000 \mathrm{rpm}$. A constant vertical force $F_{y}=-1000 \mathrm{~N}$ is applied on the shaft at bearing level and an unbalance equal to $100 \mathrm{~g} \cdot \mathrm{cm}$

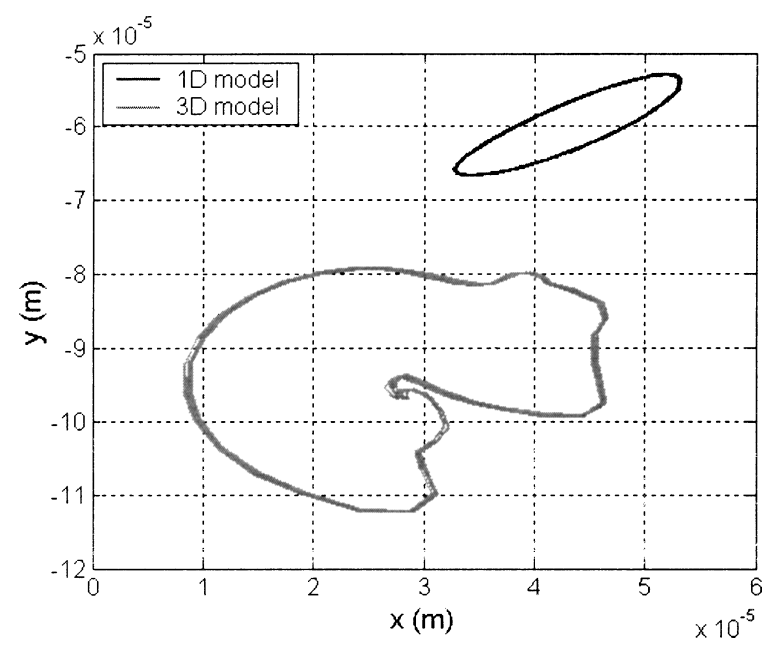

FIGURE 3

Orbit of $x-y$ displacements at bearing level $(\Omega=10,000 \mathrm{rpm})$.

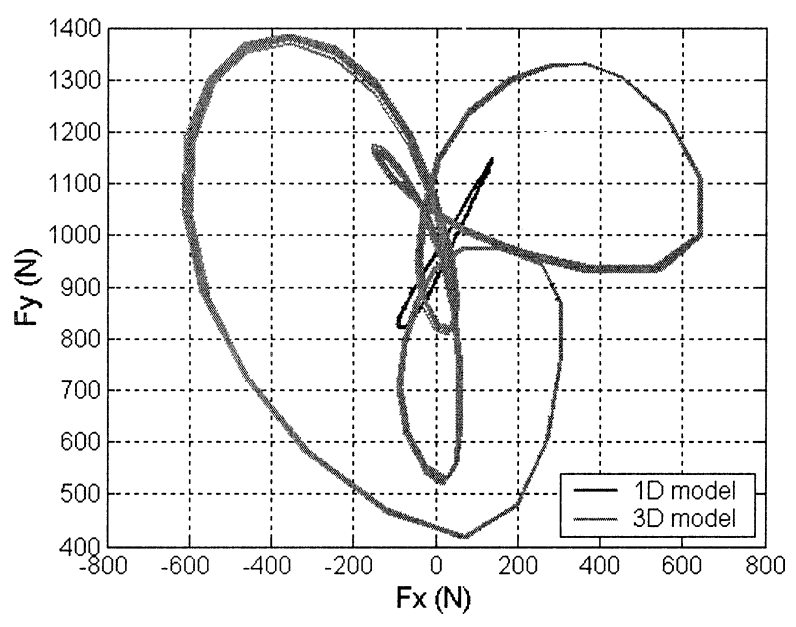

FIGURE 4

Orbit of hydrodynamic forces $(\Omega=10,000 \mathrm{rpm})$.

is applied on the disc. The structural mesh is composed of 756 isoparametric 20 nodes finite elements, thus 5472 nodes. The fluid finite difference mesh is composed of 1512 nodes.

The results obtained using the proposed approach are compared with those given by a classical rotor dynamics (1-D) approach, based on a beam finite element model of the shaft and a direct integration of fluid pressures into equivalent forces at bearing level.

The disc is supposed to be rigid for the 3-D model to get round the influence of disc flexibility on global behavior and to focus on local shaft deformations.

\section{Coupled System Behavior}

The coupled system behavior is simulated. The stabilized orbits of the mass center displacements of the shaft section at bearing level and the associated hydrodynamic forces are reported in Figures 3 and 4.

In this case, the results given by 1-D and 3-D models are significantly different and the hydrodynamic forces in the vertical direction are about three times greater for the 3-D model than for the 1-D model. These differences clearly highlight the influence of local shaft deformations on the global behavior of the rotor.

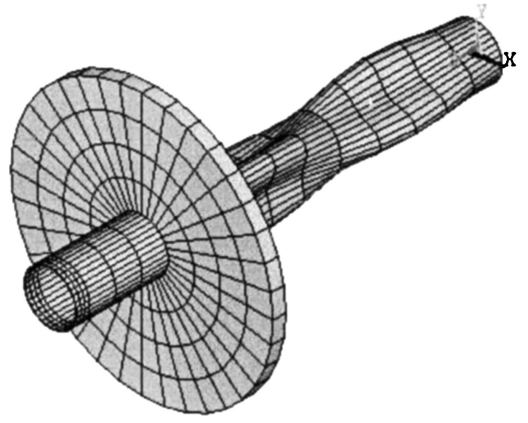

FIGURE 5

Shaft section deformation mode shape (above the disc). 


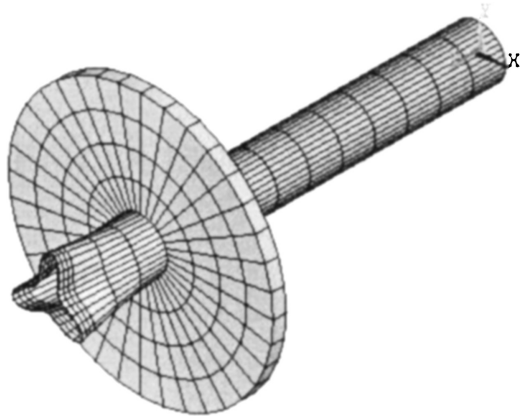

FIGURE 6

Shaft section deformation mode shape (below the disc).

\section{Influence of the Modal Basis}

The proposed approach is based on a modal decomposition of coupled displacements onto a basis composed of the first mode shapes of the non rotating undamped system in a vacuum. A convergence study showed that the 92 first mode shapes are necessary to obtain good results, inducing large computational requirements. As shown below, this computational effort can be drastically reduced by a better selection of mode shapes that have to be kept in the basis.

Mode shapes can be classified as follows: bending, torsion, and longitudinal shaft modes and ring modes involving sectional deformations of each part of the disc, which acts as a clamp (Figures 5 and 6). Considering the coupled system, it is clear that only the first global shaft bending mode shapes and the first

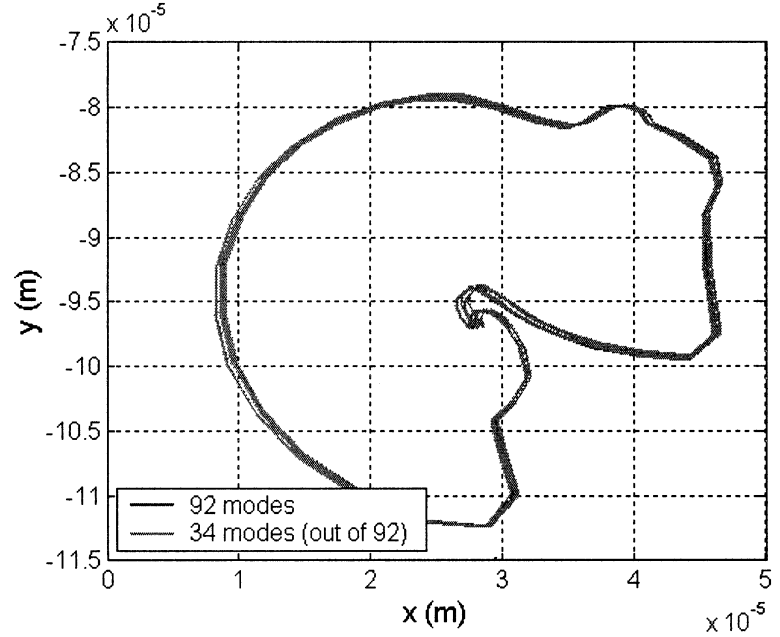

FIGURE 8

Orbit of $x-y$ displacements at bearing level $(\Omega=10,000 \mathrm{rpm})$.

ring mode shapes of the part of the shaft where the bearing is mounted are of interest.

This assumption is confirmed by the results presented in Figure 7 which gives the cumulated modal participation of the 92 first mode shapes during a full simulation:

$$
s(i)=\sum_{j}|q(i, j)|
$$

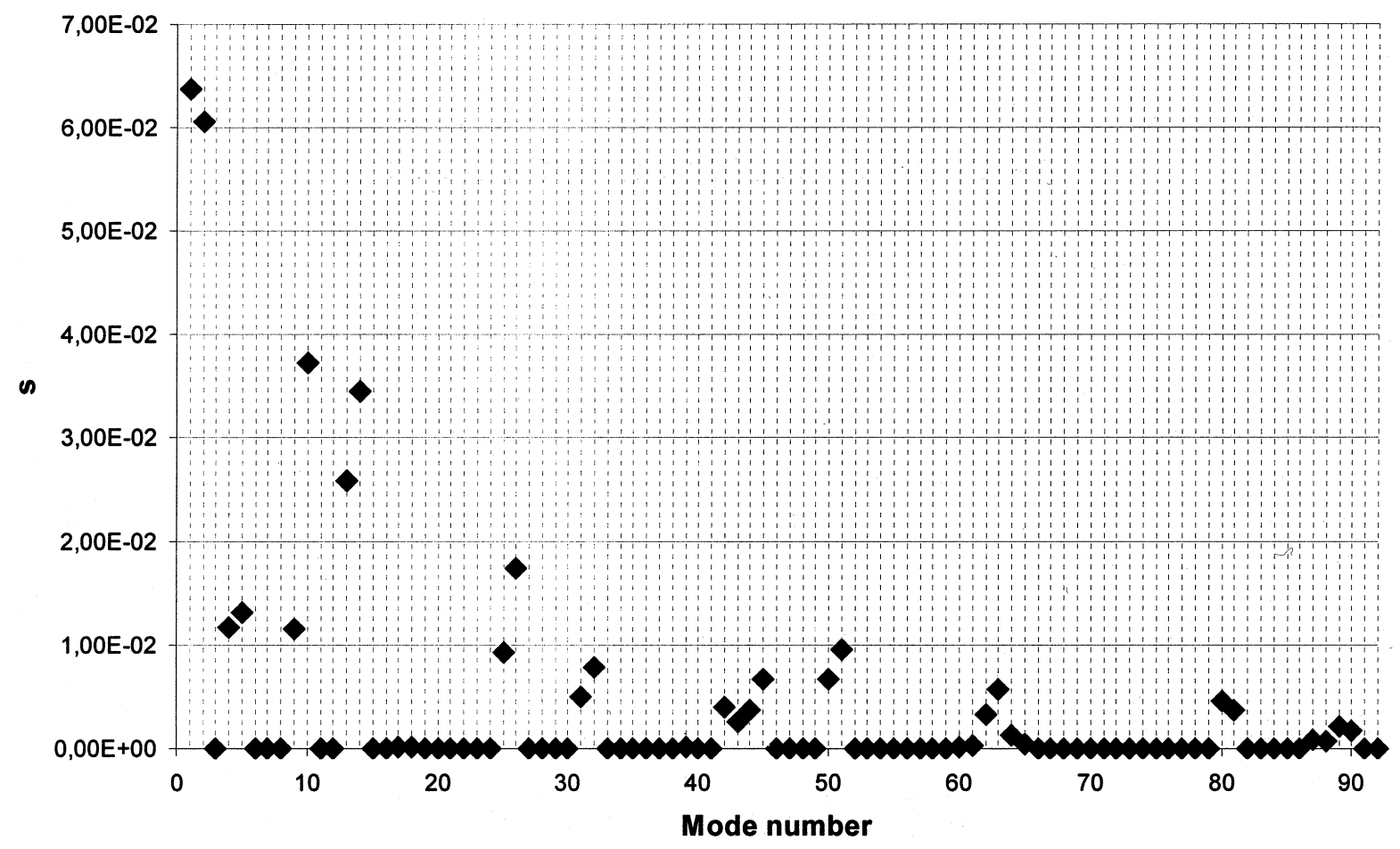

FIGURE 7

Mode participation. 


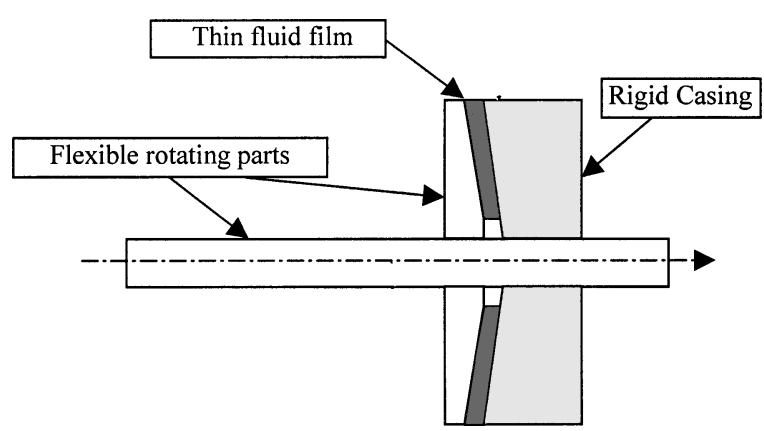

FIGURE 9

Coupled system.

where $q(i, j)$ is the value of the generalized coordinate of the $i$ th mode at time $t_{j}$.

Consequently, good selection of modes permits reducing the modal basis to 34 mode shapes without any loss of accuracy (Figure 8).

\section{Test Case $\mathbf{N}^{\circ} \mathbf{2}$}

\section{Presentation}

The coupled system considered here is composed of a wheel/shaft assembly and a fluid film clustered between the wheel and a rigid casing (Figure 9).

The shaft, made of steel, is supported at both ends and an impeller is mounted on the shaft at $60 \%$ of its length. Its main characteristics are: length $L=0.25 \mathrm{~m}$ and radius $r_{a}=0.005 \mathrm{~m}$. The wheel is composed of 2 flanges with 8 straight blades between them. Its main characteristics are: inner radius $R_{i}=0.01 \mathrm{~m}$, outer radius $R_{e}=0.05 \mathrm{~m}$, flanges and blades thickness $e=$ $0.001 \mathrm{~m}$, and blades slope versus radial direction $\beta=30^{\circ}$. A rigid hub links the shaft and the wheel.

In order to highlight the influence of local deformations on the global behavior, two models are considered: a rigid wheel model (RW) where the wheel material characteristics are set to $\rho=7800 \mathrm{~kg} / \mathrm{m}^{3}$ and $E=2.10^{15} \mathrm{~Pa}$ and a flexible wheel model (FW) where the wheel material characteristics are set to $\rho=$ $7800 \mathrm{~kg} / \mathrm{m}^{3}$ and $E=2.10^{11} \mathrm{~Pa}$.

The mechanical forces applied on the system are a vertical constant force $F_{Y}=-50 \mathrm{~N}$ and an unbalance of $1 \mathrm{~g} \cdot \mathrm{cm}$, both

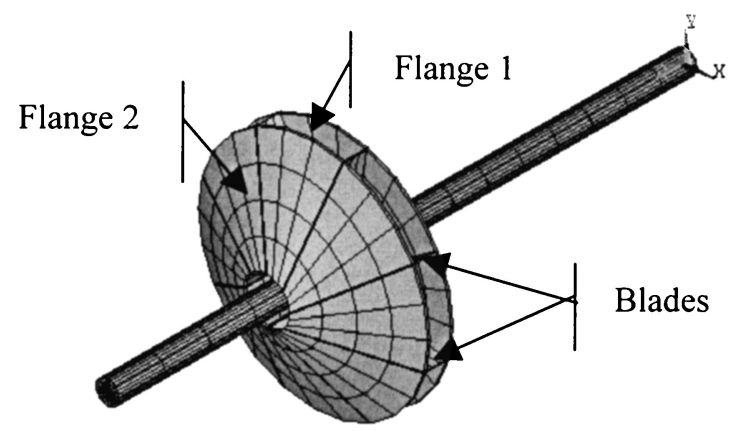

FIGURE 10

Structural mesh.

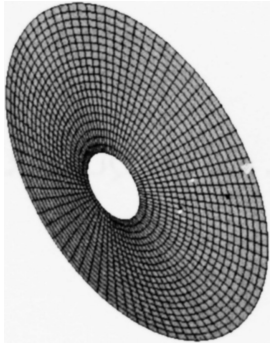

FIGURE 11

Fluid mesh.

applied at wheel level. The structure is discretized using 888 finite elements and 6363 nodes (Figure 10). The fluid film is located between a flange and the casing. Its thickness is $\mathrm{C}=$ $0.2 \mathrm{~mm}$ and its dynamic viscosity is $\mu=0.01 \mathrm{~Pa}$.s. The associated finite differences mesh is composed of 1600 nodes (Figure 11).

\section{Comparisons Between RW and FW Models}

The evolution of the frequencies versus the speed of rotation are reported in the Campbell diagram, presented in Figure 12, for the first three bending modes of the isolated structure for both RW and FW models. If the influence of wheel flexibility is negligible for the first mode, wheel/shaft coupling has great importance for modes 2 and 3.

For a rotational speed $\Omega=10000 \mathrm{rpm}$, the main characteristics of the coupled system's behavior are quantified in Table 1 which gives the displacements $(x, y, z)$ of the shaft center at wheel level and the associated hydrodynamic forces $\left(F_{X}, F_{Y}\right.$, $F_{Z}$ ). The orbit corresponding to the unbalanced response is reported Figure 13.

The behavior given by RW and FW models is significantly different. The rigid wheel hypothesis underestimates shaft bending. The deformed shape of the structure (FW model) is presented in Figure 14. Figure 15 shows the axial displacements

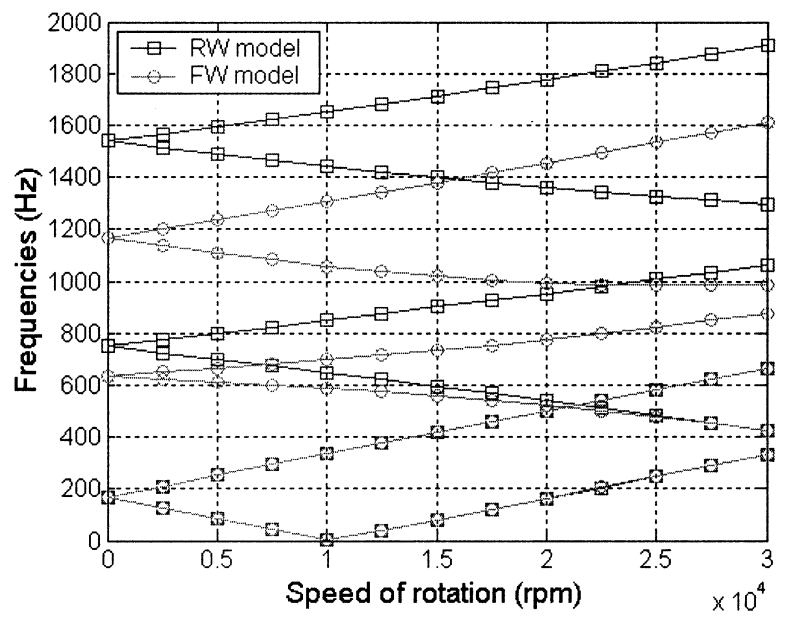

FIGURE 12

Campbell diagram. 
TABLE 1

Influence of wheel flexibility $(\Omega=10,000 \mathrm{rpm})$

\begin{tabular}{lrrr}
\hline & \multicolumn{1}{c}{ RW } & \multicolumn{1}{c}{ FW } & \multicolumn{1}{c}{$\Delta(\%)$} \\
\hline$x(\mu \mathrm{m})$ & 70.3 & 50.8 & -27.8 \\
$y(\mu \mathrm{m})$ & -174.7 & -208.9 & 19.6 \\
$z(\mu \mathrm{m})$ & -1.5 & -1.4 & -6.7 \\
$F_{X}(\mathrm{~N})$ & 7.0 & 5.2 & -25.7 \\
$F_{Y}(\mathrm{~N})$ & 17.0 & 13.8 & -18.8 \\
$F_{Z}(\mathrm{~N})$ & -110.3 & -92.8 & -15.9 \\
\hline
\end{tabular}

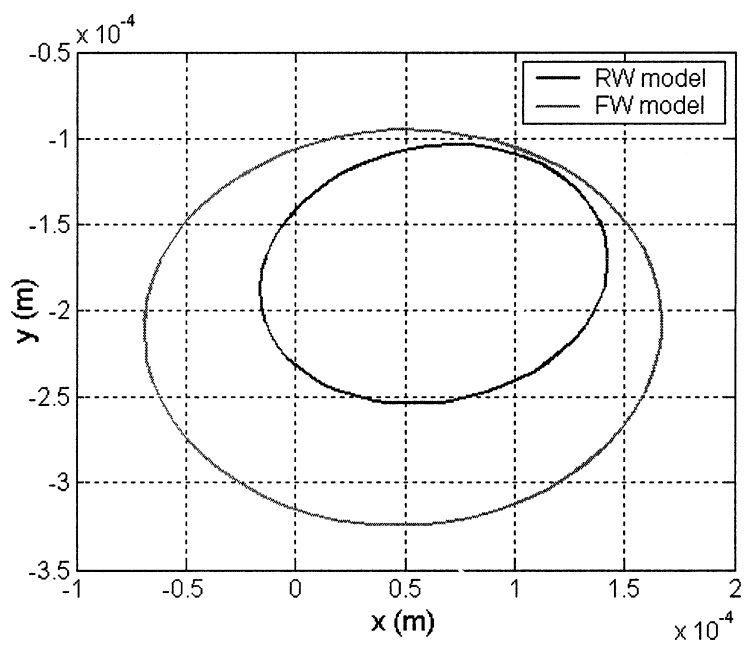

FIGURE 13

Orbit of $x-y$ displacements at wheel level $(\Omega=10,000 \mathrm{rpm})$.

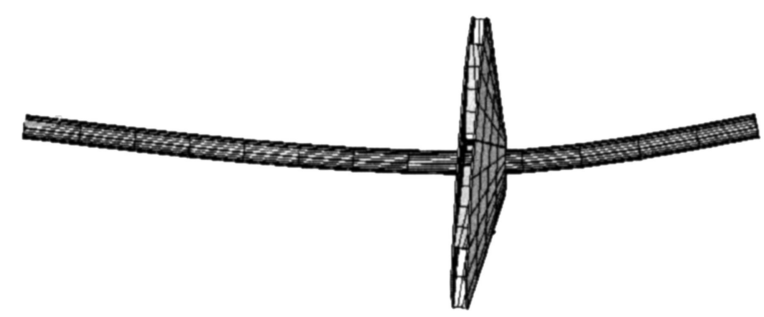

FIGURE 14

Deformed shape of wheel shaft assembly (FW model).

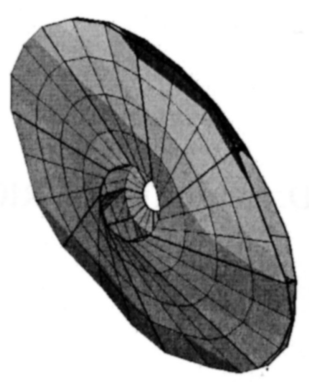

FIGURE 15

Axial displacements of the wheel (FW model). for the FW model, highlighting the local deformations due to the fluid.

\section{CONCLUSIONS}

Rotating parts of turbomachines are studied classically using uncoupled approaches that focus on specific parts and phenomena. To improve machine capabilities, it is increasingly necessary to predict the global behavior of shaft lines. For example, surrounding fluids or couplings between flexible parts of the machine must be taken into account accurately.

The procedure proposed is based on a global 3-D model of a coupled fluid-wheel-shaft assembly. Its major interest is that it combines modal reduction with an interface grid concept, permitting significant reduction of computational effort and leading to efficient coupling between fluid and structural models developed independently by using existing available tools.

The procedure is first applied to a thin-walled shaft/disc assembly mounted on a hydrodynamic bearing. This simple and well-known configuration shows the influence of local shaft deformations on the global behavior and illustrates an optimized choice of the mode shapes chosen. The second test case deals with an academic but more realistic structure composed of a wheel/shaft assembly coupled with a fluid film clustered between the wheel and a rigid casing. The influences of fluid and local deformations are clearly highlighted.

\section{REFERENCES}

Baskharone, E. A., and Hensel, S. J. 1991. A finite element perturbation approach to fluid/rotor interaction in turbomachinery elements (Part 1: Theory; Part 2: Application). ASME Journal of Fluids Engineering 113:353-361.

Bendiksen, O. O. 2000. Transonic bending flutter in rotors and cascades, in P. Ferrand and S. Aubert (Eds.). Proceedings of the 9th International Symposium on Unsteady Aerodynamics, Aeroacoustics and Aeroelasticity of Turbomachines, Grenoble: Presses Universitaires de Grenoble, 791-802, Lyon.

Berger, S., Bonneau, O., and Frene, J. 2000. Influence of a levelness defect in a thrust bearing of the dynamic behavior of an elastic shaft. Journal of Sound and Vibration 249(1):41-53.

Berlioz, A., Der Hagopian, J., Dufour, R., and Draoui, E. 1996. Dynamic behavior of a drill-string: Experimental investigation of lateral instabilities. ASME Journal of Vibration and Acoustics 118:292-298.

Childs, D. W. 1991. Fluid-structure interaction forces at a pumpimpeller-force axial analysis. ASME Journal of Vibration and Acoustics 113(1):108-115.

Desbordes, H., Fillon, M., Chan Hew Wai, C., and Frene, J. 1994. Dynamic analysis of tilting-pad journal bearing-Influence of pad deformations. ASME Journal of Tribology 116:621-628.

Frene, J., Nicolas, D., Degueurce, B., Berthe, D., and Godet, M. 1990. Lubrification hydrodynamique - Paliers et butées. Paris: Eyrolles.

Irretier, H., Jacquet-Richardet, G., and Reuter, F. 1999. Numerical and experimental investigations of coupling effects in anisotropic elastic rotors. International Journal of Rotating Machninery 5(4):263-271.

Iwatsubo, T., Sheng, B. C., and Ono, M. 1990. Experiment of static and dynamic characteristics of spiral grooved seals. 6th Workshop Rotordynamic Instability Problems in High Performance 
Turbomachinery, NASA Conference Publication $n^{\circ} 3122$, College Station, Texas.

Jacquet-Richardet, G., Ferraris, G., and Rieutord, P. 1996. Frequencies and modes of rotating flexible bladed disc-shaft assemblies: a global cyclic symmetry approach. Journal of Sound and Vibration 191(5):901-915.

Lornage, D., Chatelet, E., and Jacquet-Richardet, G. 2001. Effects of wheel-shaft-fluid coupling and local wheel deformations on the global behavior of shaft lines. ASME Paper 2001-GT-233.

Moyroud, F., Cosme, N., Jocker, M., Fransson, T. H., Lornage, D., and Jacquet-Richardet, G. 2000. A fluid-structure interfacing tech- nique for computational aeroelastic simulations, in P. Ferrand and S. Aubert (Eds.). Proceedings of the 9th International Symposium on Unsteady Aerodynamics, Aeroacoustics and Aeroelasticity of Turbomachines, Grenoble: Presses Universitaires de Grenoble, 721-738, Lyon.

Moyroud, F. 1998. Fluid-Structure Integrated Computational Methods for Turbomachinery Blade Flutter and Forced Response Predictions. Thèse de doctorat, INSA de Lyon (98 ISAL 0101), PhD thesis, KTH Stockholm (TRITA-KRV-1998-2).

Renshaw, A. A. 1998. Critical speed for floppy disks. Journal of Applied Mechanics 65:116-120. 

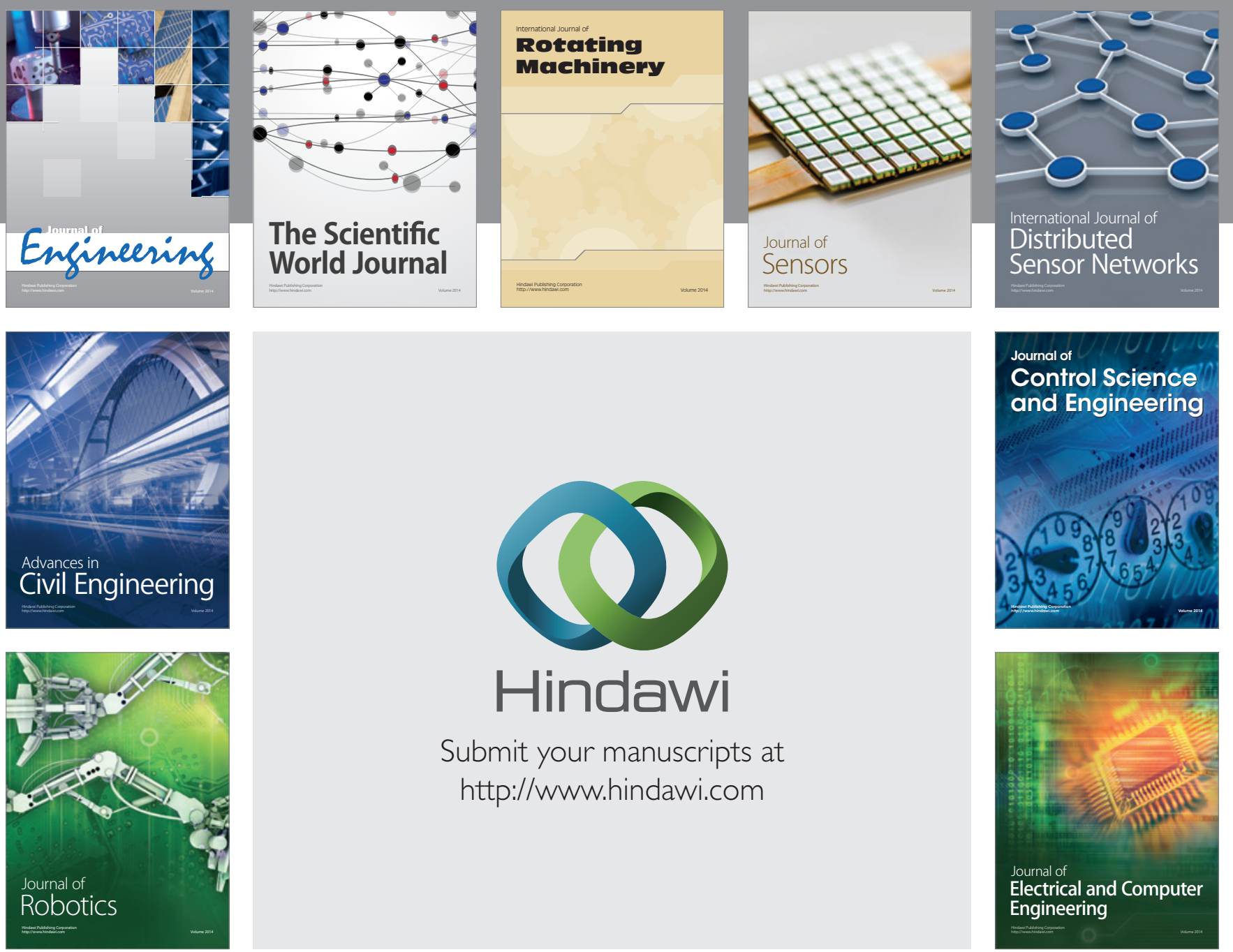

Submit your manuscripts at

http://www.hindawi.com
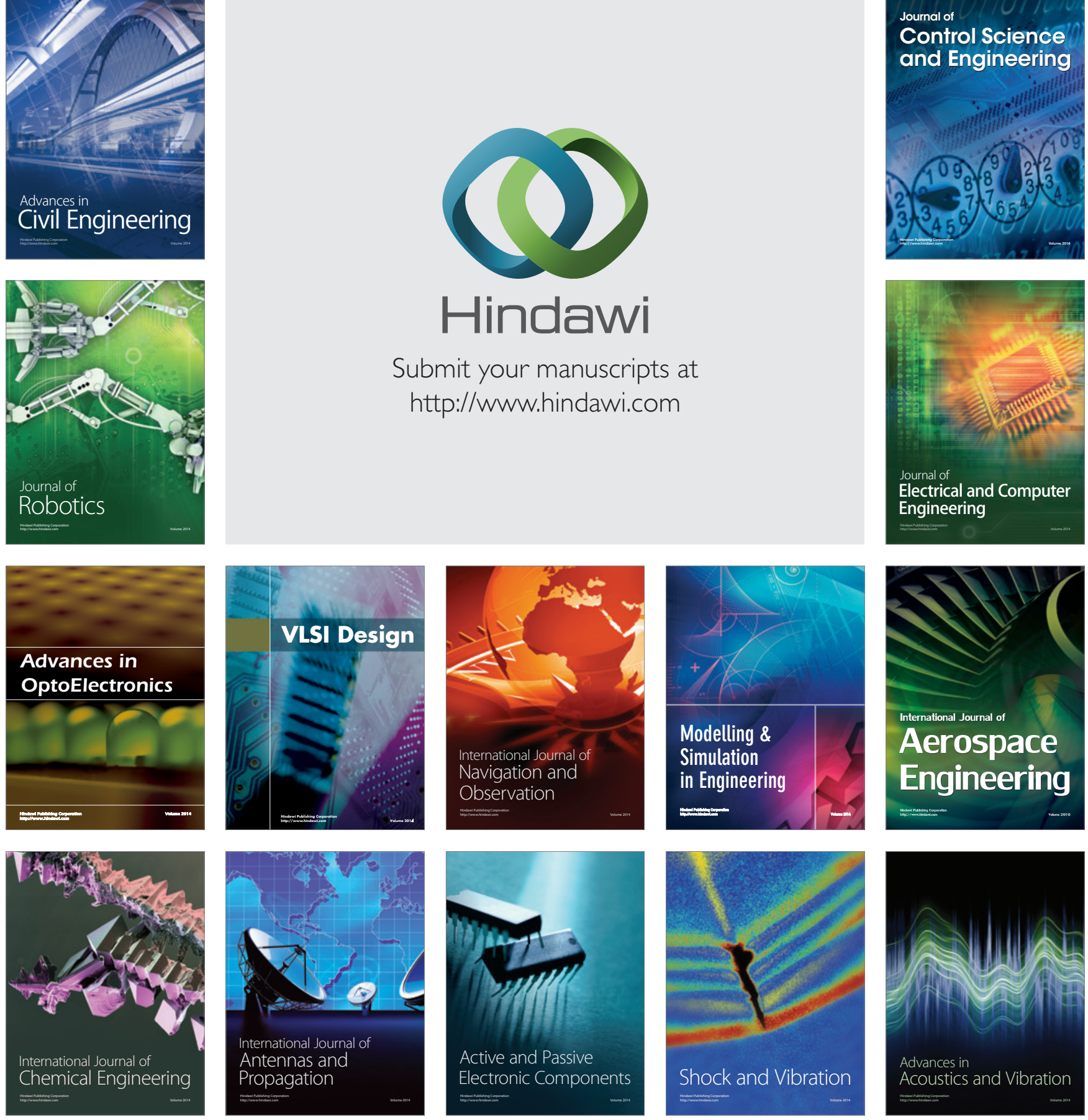